\title{
ARTICLE
}

\section{A rapid dehydration leaf assay reveals stomatal response differences in grapevine genotypes}

\author{
Daniel W Hopper, Ryan Ghan and Grant R Cramer
}

A simple and reliable way of phenotyping plant responses to dehydration was developed. Fully-developed leaves were detached and placed in a closed plastic box containing a salt solution to control the atmospheric water potential in the container. Three hours of dehydration (weight loss of the leaf) was optimal for measuring changes in stomatal response to dehydration. Application of the plant hormone abscisic acid (ABA) prior to leaf detachment decreased the amount of water loss, indicating that the assay was able to detect differences based on a stomatal response to dehydration. Five different Vitis genotypes (V. riparia, V. champinii, V. vinifera cv. Shiraz, V. vinifera cv. Grenache and $V$. vinifera cv. Cabernet Sauvignon) with known differences in drought tolerance were screened for their dehydration response and the results obtained corresponded to previous reports of stomatal responses in the vineyard. Significant differences in stomatal density along with differences in the amount and rate of water lost indicate differences in dehydration sensitivity among the genotypes screened. Differences in stomatal response to ABA were also detected. Shiraz had the lowest stomatal density and the highest ABA sensitivity among the genotypes screened, yet Shiraz lost the most amount of water, indicating that it was the least sensitive to dehydration. Despite having the highest stomatal density and intermediate stomatal sensitivity to ABA, $V$. riparia lost the smallest amount of water, indicating that it was the most sensitive to dehydration. The assay presented here represents a simple and reliable phenotyping method for plant responses to leaf dehydration.

Horticulture Research (2014) 1, 2; doi:10.1038/hortres.2014.2; published online 22 January 2014

\section{INTRODUCTION}

A fundamental response to drought by plants is to conserve water through stomatal regulation. Stomata are pores found in the epidermis of the leaf that allow for an influx of $\mathrm{CO}_{2}$ for photosynthesis and consequently water loss through transpiration. These pores are flanked by specialized cells, known as guard cells. Within these guard cells, the plant hormone abscisic acid (ABA) is one of the most important chemical signals that triggers a signaling cascade leading to stomatal closure under abiotic conditions such as drought. Guard cell control of transpiration and water loss is strongly associated with drought tolerance. ${ }^{1,2}$

There are large differences in drought tolerance among grapevine cultivars and species. ${ }^{3-5}$ Previously, Vitis vinifera L. cv. Shiraz (also known as Syrah) and Grenache have been classified as anisohydric and isohydric, respectively. ${ }^{6}$ These two different classifications have been around for over half a century. ${ }^{7}$ Genotypes with anisohydric characteristics have decreasing leaf or stem water potentials with decreasing soil water potentials and maintain maximum photosynthesis $\left(A_{\max }\right)$ and stomatal conductance $\left(g_{\max }\right)$ even at low leaf water potentials $\left(\psi_{\text {leaf }}\right)$. Isohydric genotypes, like Grenache, keep their leaf water potentials above that of soil water potentials by closing their stomata quickly in response to water deficit, consequently decreasing photosynthesis as well as transpiration. ${ }^{6}$ Most of the grape Vitis species in general can be considered anisohydric with exceptions as stated above, as well as Cabernet Sauvignon which has recently been shown to have isohydric characteristics. ${ }^{8,9}$ This phenomenon also occurs in other plant species. For example, sunflower and barley show low stomatal response and are classified as anisohydric while maize and poplar have more stomatal control at low $\psi_{\text {leaf }}$ and are classified as having isohydric behavior. ${ }^{10}$ However, it has been shown that under different conditions or different parts of the season, the same genotype may display characteristics of both isohydric and anisohydric behaviors, making it difficult to interpret results, and therefore, the term near iso/anisohydric is often used to describe different genotypes. $^{3,8}$

In addition to the Vitis vinifera cultivars mentioned above, two other North American Vitis species were selected in this study due to their differences in drought tolerance. They are frequently used as rootstocks and are commonly known as Ramsey (Vitis champinii, a naturally occurring hybrid between Vitis candicans and Vitis rupestris) and Riparia Gloire (Vitis riparia). Ramsey originates from the hot, dry regions of Texas and is reported to be drought tolerant, while Riparia Gloire originates from wet, riparian areas and is reported to be drought sensitive. ${ }^{5,11}$ Rootstocks are used routinely in viticulture for the properties they confer to a scion or the fruit bearing portion of the plant, such as vigor and drought resistance, as well as a resistance to the grape insect pest, phylloxera (Daktulosphaira vitifoliae), making them vital in most wine growing regions. ${ }^{11}$ Few studies $^{5}$ have investigated the dehydration response at the leaf level of these two genotypes, but have focused on their role as a rootstock in their relationship to a scion.

Phenotyping grapevines for drought tolerance in the field requires many years for vine establishment and is influenced significantly by soil and climate variability. In order to improve the assessment of dehydration responses among different grapevine genotypes in a highly controlled manner, a rapid dehydration assay was developed and tested. The response of leaves in this rapid dehydration for different genotypes was consistent with field observations and known physiological responses to ABA. It appears to be a promising method for screening grapes for differences in water use. 


\section{MATERIALS AND METHODS}

\section{Plant material and growth conditions}

Vines of five grape genotypes (Vitis vinifera cv. Cabernet Sauvignon clone 8 Vitis vinifera cv. Grenache Noir clone 2, Vitis vinifera cv. Shiraz clone 7, Vitis riparia cv. Riparia Gloire and Vitis champinii cv. Ramsey (a naturally occurring hybrid between Vitis candicans and Vitis rupestris) were grown in $13.3 \mathrm{~L}$ pots containing approximately $10 \mathrm{~L}$ SuperSoil @ (ScottsMiracle-Gro, Marysville, $\mathrm{OH}, \mathrm{USA}$ ) potting mix supplemented with Ultragreen Tomato and Vegetable Food (Pennington Seed Corp., Madison, GA, USA) slow release fertilizer (510-10). Plants were grown in a glass greenhouse with supplemental sodium vapor lamp lighting ( $16 \mathrm{~h}$ light (minimum $400 \mu \mathrm{mol} \mathrm{m} \mathrm{m}^{-2} \mathrm{~s}^{-1}$ ) at $28{ }^{\circ} \mathrm{C}$ and $8 \mathrm{~h}$ dark at $18{ }^{\circ} \mathrm{C}$ cycle). Vines were irrigated twice a week with tap water.

\section{Leaf selection}

Fully developed leaves for each genotype were selected based on leaf length measurements taken from the first expanded leaf distal to the growing shoot tip down the length of the shoot. Leaf length measurements were taken from the petiole to the tip of the leaf down the midvein. A leaf was classified as 'fully developed' at the node where leaf length no longer increased in size. Fully developed leaves were used for the dehydration assays unless otherwise specified. These leaves were also at their maximal photosynthetic rates (data not shown). Gas exchange measurements were also used to select leaves with good photosynthesis and conductance values in order to try to reduce variability and eliminate unusual leaves that were not functioning normally.

\section{Gas exchange measurements}

In order to ensure that observations seen were not due to variation in leaf photosynthesis $\left(A_{\max }\right)$ and stomatal conductance $\left(g_{\mathrm{s}}\right)$, gas exchange measurements were taken with a LI-6400XT portable photosynthesis system (LiCor Inc., Lincoln, NE, USA) using the following settings: $\mathrm{CO}_{2}$ reference, $400 \mathrm{ppm}$; light, $1100 \mu \mathrm{mol} \mathrm{m}{ }^{-2} \mathrm{~s}^{-1}$; leaf temperature, $27{ }^{\circ} \mathrm{C}$; flow rate, $400 \mu \mathrm{mol} \mathrm{s}{ }^{-1}$.

\section{Leaf dehydration assay}

A leaf was detached from the vine (with most of the petiole removed) and dehydrated by placing immediately in an airtight plastic box $(1.2 \mathrm{~L}$ Rubbermaid Takealong (c) container, Newell Rubbermaid, Atlanta, GA, USA) containing $50 \mathrm{~mL}$ of a $333 \mathrm{mM} \mathrm{NaCl}$ solution. Several hours before the start of the assay, the salt solution was placed in the container to provide a constant relative humidity (water potential), providing a tightly regulated environment for leaf water loss. A single leaf was placed on a custom-made chicken-wire platform within the plastic container with the abaxial side down and above the $\mathrm{NaCl}$ solution. The lid to the container was then securely fastened within a couple of $\mathrm{s}$ after excision. Within $15 \mathrm{~s}$ after excision, the leaf was removed and weighed; the weighing procedure took about $5 \mathrm{~s}$. The leaf was placed back into the box and placed inside an AR-75 Percival Scientific growth chamber (Percival Scientific, Boone, IA, USA) with constant fluorescent-incandescent light $\left(200 \mu \mathrm{mol} \mathrm{m} \mathrm{m}^{-2} \mathrm{~s}^{-1}\right)$ and temperature $\left(27^{\circ} \mathrm{C}\right)$. The leaf was removed from the dehydration box and weighed to the nearest $\mu \mathrm{g}$ on a scale every $30 \mathrm{~min}$ thereafter and immediately placed back into the box, which was re-sealed and placed back into the growth chamber until the next measurement. In this way, 20 samples (e.g., 5 genotypes $\times 4$ replicates) could be measured in a sequential manner every $1.5 \mathrm{~min}$ within the 30-min intervals of an individual experiment. Care was taken not to spill any salt solution on the leaves.

\section{Stomatal density determination}

For each genotype, a fully developed leaf similar to the ones used for the dehydration assays from three different plants was used. Clear nail polish was applied to three different areas of each leaf avoiding the midvein and allowed to dry. Clear packing tape was then used to peel off the nail polish, which was then placed onto a plain microscope slide. Images were captured with a digital camera (Orca-ER; Hamamatsu Phontonics K.K., Hamamatsu, Japan, http:// www.hamamatsu.com) controlled by SimplePCl software (Hamamatsu Phontonics K.K.). Stomata were counted using ImageJ (National Institutes of Health, Bethesda, MD, USA) (1.47v) with the cell counter plugin. ${ }^{12}$

\section{Leaf area calculation}

Leaves from multiple Cabernet Sauvignon shoots grown in conditions stated above were harvested and detached from their petioles. Leaves encompassing immature to fully developed leaves were used. The distance from the petiole to the tip of the leaf down the midvein was measured. Detached leaves were then arranged on a white background with a ruler placed on one edge for scale assignment, and then photographed with a Canon Rebel T2i digital camera (Canon Inc., Ohta-ku, Tokyo, Japan). Leaf images were imported into ImageJ to determine leaf area. ${ }^{12}$ Briefly, color images were first converted to an 8-bit grayscale, drawing a line over a known distance on the photographed ruler, and then globally assigning this distance using the Set Scale command to assign the measurement scale of each image. The leaf images were further processed into binary images using the Make Binary command. Using the rectangular selection tool, each leaf was surrounded before calculating the area with the Analyze Particles command. A polynomial second order equation, $y=931.2+(-44.4) x+1.292 x^{2}$, was calculated using GraphPad Prism (C) V4.0 (GraphPad Software Inc., La Jolla, CA, USA) to fit a curved line to the plotted leaf area and leaf length measurements.

\section{ABA application}

(+)-ABA (TCl America, Tokyo Chemical Industry Co., Ltd., Chuo-ku, Tokyo, Japan, http://www.tcichemicals.com/en/us/) was sprayed onto fully developed intact leaves of potted Cabernet Sauvignon vines. Different leaves were treated with $1,10,100$ or $500 \mu \mathrm{M} A B A$. ABA concentrations were made from a $1 \mathrm{mM} \mathrm{ABA}$ stock solution after $A B A$ had been dissolved in $1 \mathrm{~mL}$ of methanol and then brought up to $1 \mathrm{~L}$ with distilled-deionized water. Final concentrations were made by serial dilution and $0.1 \%$ Triton- $X$ was added as a surfactant to insure good spray coverage on the leaf. The control solution included methanol and $0.1 \%$ Triton- $\mathrm{X}$ at the same concentrations while omitting the $A B A$. Gas exchange measurements were taken for $4 \mathrm{~h}$ after application of $A B A$, and then leaves were excised and placed in the dehydration boxes and subjected to a 3-h dehydration assay.

\section{Stomatal sensitivity to $A B A$}

Stomatal conductance was measured over time with the LI-6400XT portable photosynthesis system using the settings described above. ABA treatments $(0,100$ and $500 \mu \mathrm{M})$ were applied to both sides of intact leaves until fully wet and the effect on stomatal conductance was measured over time for all five genotypes. A set of three replicates was run in one experiment and three experiments were performed on separate days. Experimental measurements were made at mid-day starting at 10 a.m. and finishing near 2 p.m. Sprays and stomatal conductance measurements were conducted in sequential order like the leaf dehydration assay.

\section{Statistical analysis}

Data are presented as the mean \pm standard error of the mean (s.e.m.). Graphs were generated and analyzed using GraphPad Prism @ $)$. Statistical significance of the data was evaluated using two-way repeated measure analysis of variance (ANOVA) and Bonferroni post-tests.

\section{RESULTS}

A good screen for phenotyping plants should use methods that are relatively simple and fast. A leaf dehydration assay was developed with this intent. Leaves were excised and placed into a plastic box containing a salt solution. The leaves were weighed over time to measure the amount of water loss (see the section on 'Materials and methods'). In an initial test of the system, Cabernet Sauvignon leaves were treated for $8 \mathrm{~h}$ of dehydration (Figure 1). The response was multiphasic with a near linear relationship in the beginning of water loss with time. A linear regression was plotted for the first hour and a half and had an $r^{2}$ of 0.91 and a slope of 0.0012 (dotted line in Figure 1). Subsequently, water loss diminished with a decaylike function over the course of $3 \mathrm{~h}$, eventually reaching another linear phase for the rest of the $8 \mathrm{~h}$. It was determined that the first 3-h time window was optimal for detecting changes in water loss from the leaf and this time window was used for all further experiments. A major assumption is that water loss is occurring primarily through the stomata and that they are responding by closing during the dehydration event. Other sources of water loss through the cuticle or cut end of the petiole were considered to be negligible.

To test if water loss from the leaves was primarily a response of stomata to dehydration, $A B A$, a known elicitor of stomatal closure, 


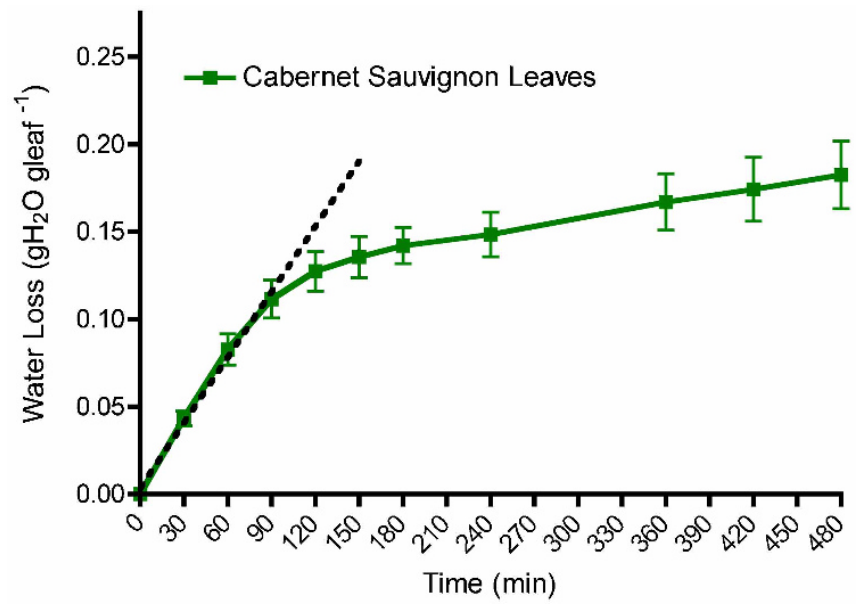

Figure 1. Water loss of Cabernet Sauvignon leaves over an 8-h period. Intermediate leaves were selected based on their position from the shoot tip (fifth to sixth leaf). Water loss for the first hour and a half follows a linear trend followed by a decrease in the amount of water lost. Each data point is presented as the mean \pm s.e.m. $(n=4)$. The dotted line was fitted to the first three time points by linear regression.

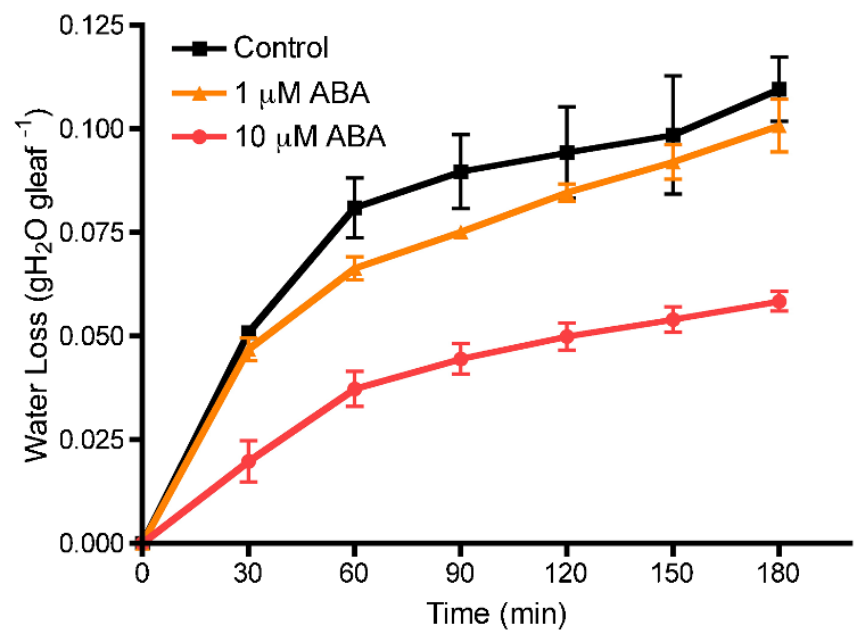

Figure 2. The effect of a 4-h treatment of ABA on water loss in the leaf dehydration assay. Each data point is presented as the mean \pm s.e.m. Control $(n=2) ; 1 \mu \mathrm{M}$ and $10 \mu \mathrm{M} \mathrm{ABA}(n=3)$.

was applied (Figure 2). Intact leaves were sprayed with different concentrations of ABA. The dehydration assay was performed after $4 \mathrm{~h}$ of $A B A$ treatment, when gas exchange measurements indicated that conditions were at steady state. Application of ABA significantly affected the amount of water lost $(p \leqslant 0.01)$ (Supplementary Fig. 1). The slope of the first hour of water loss is lower at $10 \mu \mathrm{M}$ ABA (0.0006) compared to both control (0.0013) and $1 \mu \mathrm{M}$ $A B A(0.0011)$ (lines not shown). It also decreases significantly the overall amount of water loss in a 3-h period, indicating that water loss is affected by stomatal responses.

Leaf age is another factor that may influence the amount of water that is lost due to differences in stomatal sensitivity. ${ }^{13}$ Leaf age significantly affected $(p \leqslant 0.05)$ water loss over 3 h of dehydration (Figure 3). Young leaves (third to fourth leaf from the shoot tip) were the least responsive to dehydration and lost more water compared to intermediate (fifth to sixth leaf) or older leaves (fifteenth to sixteenth). The most responsive leaves to dehydration were within the intermediate leaf ages and fully developed.

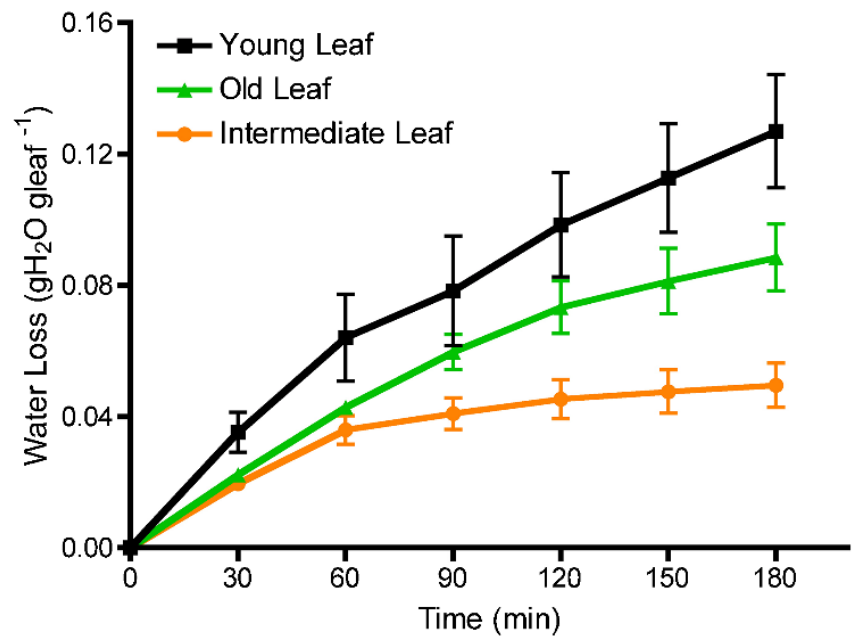

Figure 3. The effect of leaf age on water loss in the leaf dehydration assay. Green healthy leaves were selected based on their position from the shoot tip. Young leaves represented the third to fourth leaves, intermediate were the fifth to sixth leaves, and old leaves represented the fifteenth to sixteenth leaves. Each data point is presented as the mean \pm s.e.m. $(n=4)$.

It is important to measure leaf responses among multiple genotypes at the same leaf developmental stage. Fully developed leaves are easy to detect because leaf expansion has ceased. In order to determine the node at which fully developed leaves were found, leaf length (the distance from the petiole to the tip of the leaf down the midvein) from the shoot tip towards the base was measured for the five genotypes in this study (Figure 4). This was to ensure that water lost due to dehydration was not based on differences between leaf age for each genotype. Once leaf length reached a plateau and no longer significantly increased, these leaves were considered fully developed. For Riparia Gloire, this plateau occurred at node 8 with an average leaf length of $16.7 \mathrm{~cm}$, while for the other genotypes, this occurred at node 10 with an average length of 8.0, 10.7, 11.1 and $12.2 \mathrm{~cm}$ for Ramsey, Cabernet Sauvignon, Grenache, and Shiraz, respectively (Figure 4). Photosynthetic measurements

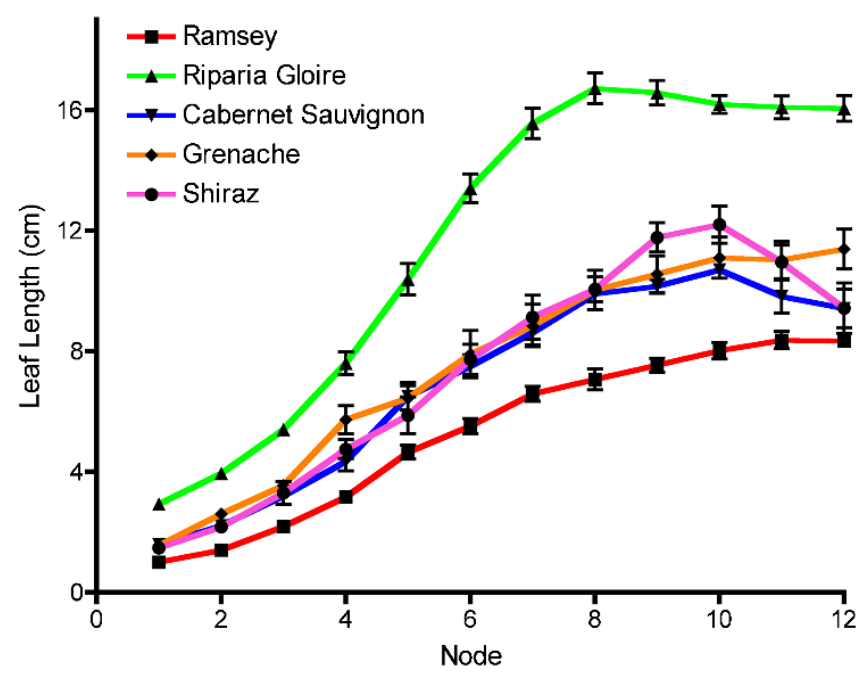

Figure 4. Determination of fully developed leaves along the shoots of five grapevine genotypes. Each data point is presented as the mean \pm s.e.m. ( $n=8$ for Grenache; $n=4$ for Shiraz; $n=6$ for Ramsey, Riparia Gloire and Cabernet Sauvignon). 
of these leaves confirmed that the selected leaves were photosynthetically optimal (data not shown).

Based on the measurements determined above, fully developed leaves were selected and used in the dehydration assay to observe differences among the genotypes based on their dehydration response (Figure 5). On a per leaf basis, Ramsey lost the smallest amount of water and Shiraz lost the most water (Figure 5a). Results from ANOVA indicated highly significant effects for time $(p \leqslant 0.0001)$, genotype $(p \leqslant 0.05)$ and their interaction $(p \leqslant 0.0001)$ (Supplementary data for Fig.2). These results were consistent with
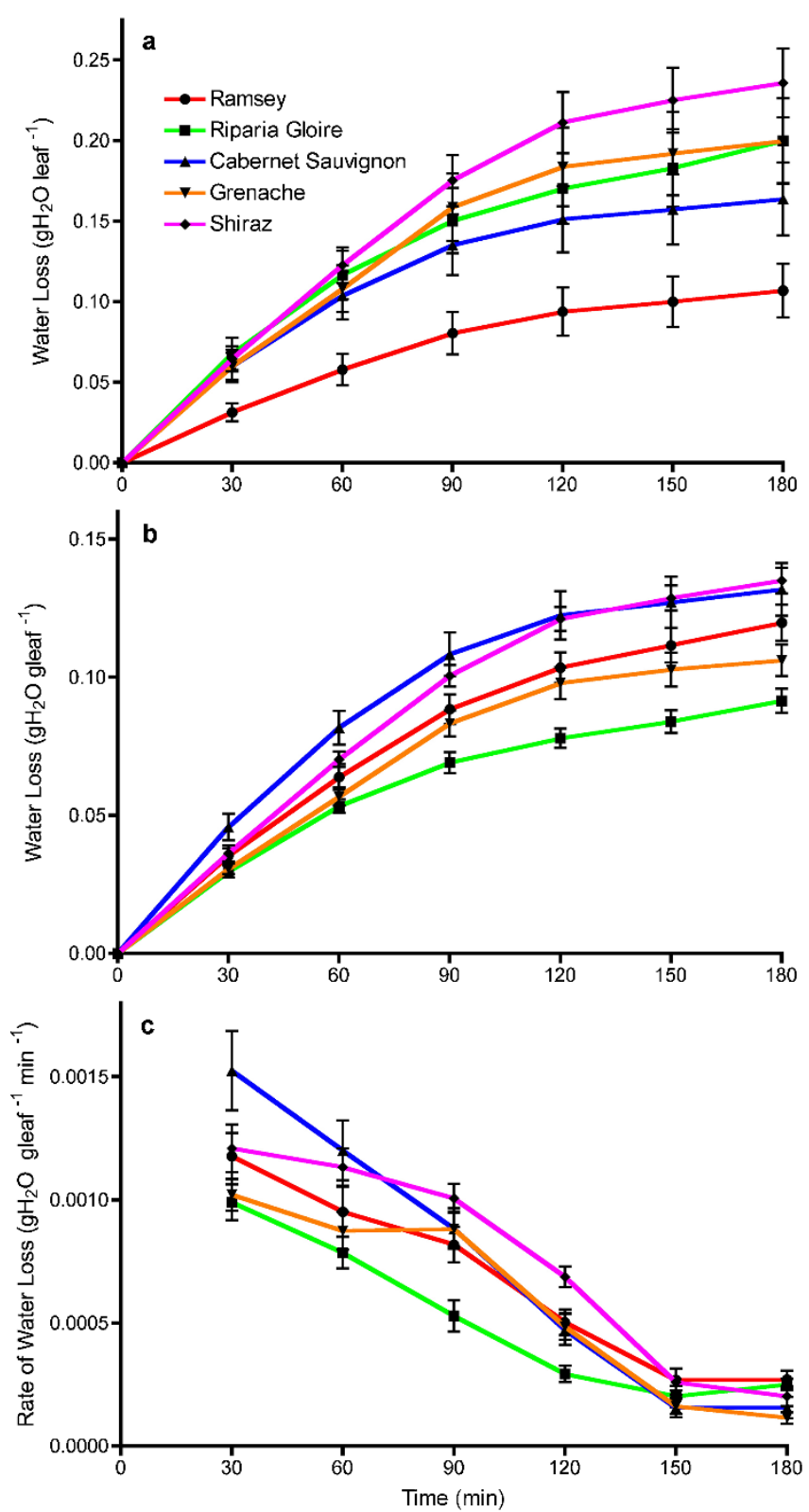

Figure 5. (a) Water loss in the dehydration assay expressed on a per leaf basis. Each data point is presented as the mean \pm s.e.m. ( $n=18$ for Ramsey, Grenache and Shiraz; $n=19$ for Riparia Gloire and Cabernet Sauvignon). (b) Water loss in the dehydration assay normalized on a leaf weight basis. Each data point is presented as the mean \pm s.e.m. ( $n=18$ for Ramsey, Grenache and Shiraz; $n=19$ for Riparia Gloire and Cabernet Sauvignon). (c) Rate of water loss from each genotype in the dehydration assay. Each data point is presented as the mean \pm s.e.m. ( $n=14$ for Ramsey; $n=18$ for Grenache and Shiraz; $n=19$ for Riparia Gloire and Cabernet Sauvignon). the difference in leaf size between these two genotypes. Leaves of Cabernet Sauvignon, Grenache and Riparia Gloire had similar amounts of water loss per leaf despite having large differences in average leaf size.

Leaf length, area and mass are other metrics that can be used to normalize the data to account for variation in leaf size. Leaf area was calculated using image analysis software and a correlation was made to leaf length (Supplementary Fig. 1). Comparing these three normalizing metrics, leaf mass was found to be the most accurate measurement with less error. Leaf area measurements have the disadvantage that it requires more time for the measurement than the other techniques and the leaf may lose more water or its metabolism may be further changed during the measurement, especially if measured with a leaf area meter. This kind of measurement could lead to additional artifacts in sensitive molecular assays of the dehydrated leaves following the dehydration assay. Leaf mass is easy to measure, is collected already at the start of the experiment and was found to be an accurate estimate of leaf size for normalizing the data. Leaf mass also had the lowest coefficient of variation (CV) for water loss; average CVs for leaf mass, length and area were $20.7 \%, 25.9 \%$ and $35.1 \%$, respectively (Supplementary Table 1). Average leaf weights ( $g$, mean \pm s.e.m., $n=8)$ for the five genotypes in ascending order were $1.75 \pm 0.17,2.01 \pm 0.10,2.20 \pm$ $0.04,2.38 \pm 0.24$ and $2.63 \pm 0.16$ for Ramsey, Cabernet Sauvignon, Shiraz, Grenache and Riparia Gloire, respectively.

A different trend was observed when the data were normalized for the amount of water lost at each time point by the leaf weight (Figure $5 b)$. ANOVA results indicated highly significant effects of genotype $(p \leqslant 0.0001)$, time $(p \leqslant 0.0001)$ and their interaction $(p \leqslant 0.0001)$ (Supplementary data for Fig. 5). Despite Riparia Gloire having the largest leaves, it lost the smallest amount of water during the 3-h leaf dehydration assay, while Shiraz and Cabernet Sauvignon lost the most water (Figure 5b). Grenache and Ramsey were intermediate and did not have significantly different water losses from each other on a leaf mass basis. The significant interaction term indicates that the genotypes varied in their water loss (stomatal response) over time or that they had different sensitivities to dehydration.

The rate of water lost over time was calculated for each genotype to elucidate these changes with time (Figure 5c). ANOVA indicated highly significant effects for time $(p \leqslant 0.0001)$, genotype $(p \leqslant 0.0001)$ and the interaction term $(p \leqslant 0.0001)$ (Supplementary data for Fig. 5B). This analysis indicated that Riparia Gloire was responding the fastest to dehydration by closing its stomata the earliest. This was also reflected in Figure $5 b$ with Riparia Gloire losing the smallest amount of water. Shiraz was shown to have the highest rate of water loss during the first 120 min of dehydration.

One possible explanation for the different rates of water loss was that the leaves from each genotype had differences in their stomatal density. If this were the case, Shiraz, which lost the most amount of water, would have the largest stomatal density of the five genotypes surveyed here and Riparia Gloire would have the lowest stomatal density. In fact, there were highly significant differences $(p \leqslant 0.01)$ among the genotypes (Figure 6; Supplementary data for Fig. 5C). Shiraz had the lowest stomatal density and Riparia Gloire had one of the highest, the exact opposite of the prediction if stomatal density was a factor. These results indicated that during the dehydration assay, Shiraz failed to respond by closing its stomata and continued to lose water. Also, despite having the largest stomatal density, Riparia Gloire had the lowest rate of water loss per leaf mass and lost the smallest amount of water, which indicated a higher sensitivity to dehydration.

Since $A B A$ is a major factor involved in stomatal response to dehydration, we tested the hypothesis that these five grapevine genotypes were different in stomatal sensitivity to $A B A$. A series of different concentrations of ABA $(1-500 \mu \mathrm{M})$ were tested in preliminary assays of stomatal conductance over time. The clearest results were detected with $100 \mu \mathrm{M}$ and $500 \mu \mathrm{M}$ ABA (Figure 7). 


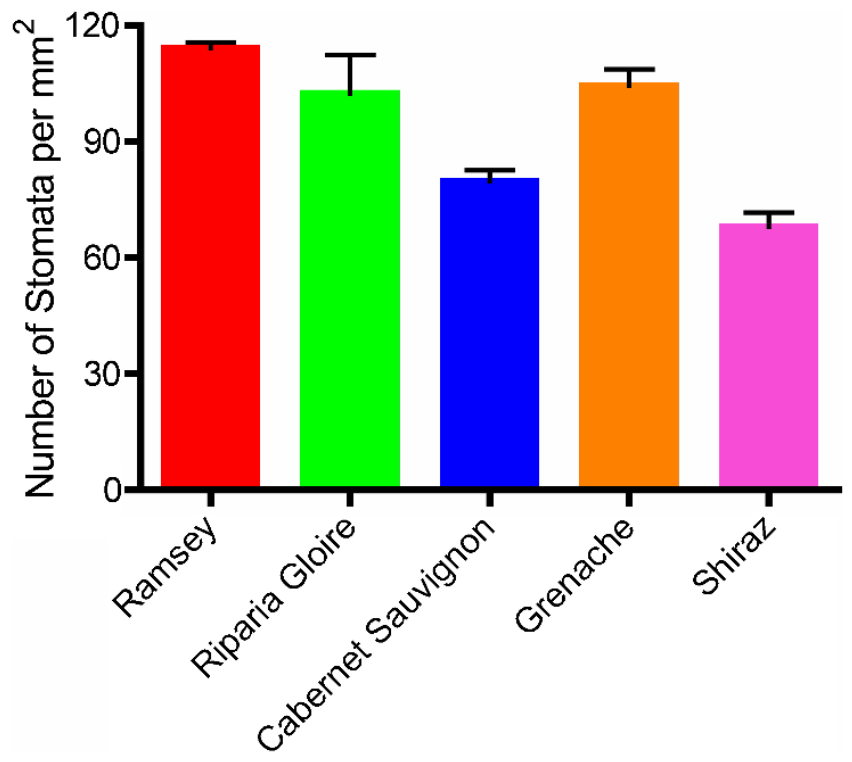

Figure 6. Stomatal density measurements of five grapevine genotypes. Data are expressed as the mean \pm s.e.m. $(n=3)$.

With $100 \mu \mathrm{M}$ ABA, water loss was decreased relative to controls over time (Figure 7a). The controls were unaffected by the control spray and thus, data were expressed as a percentage (\%) of the control. The stomatal conductance of Ramsey, Riparia and Shiraz
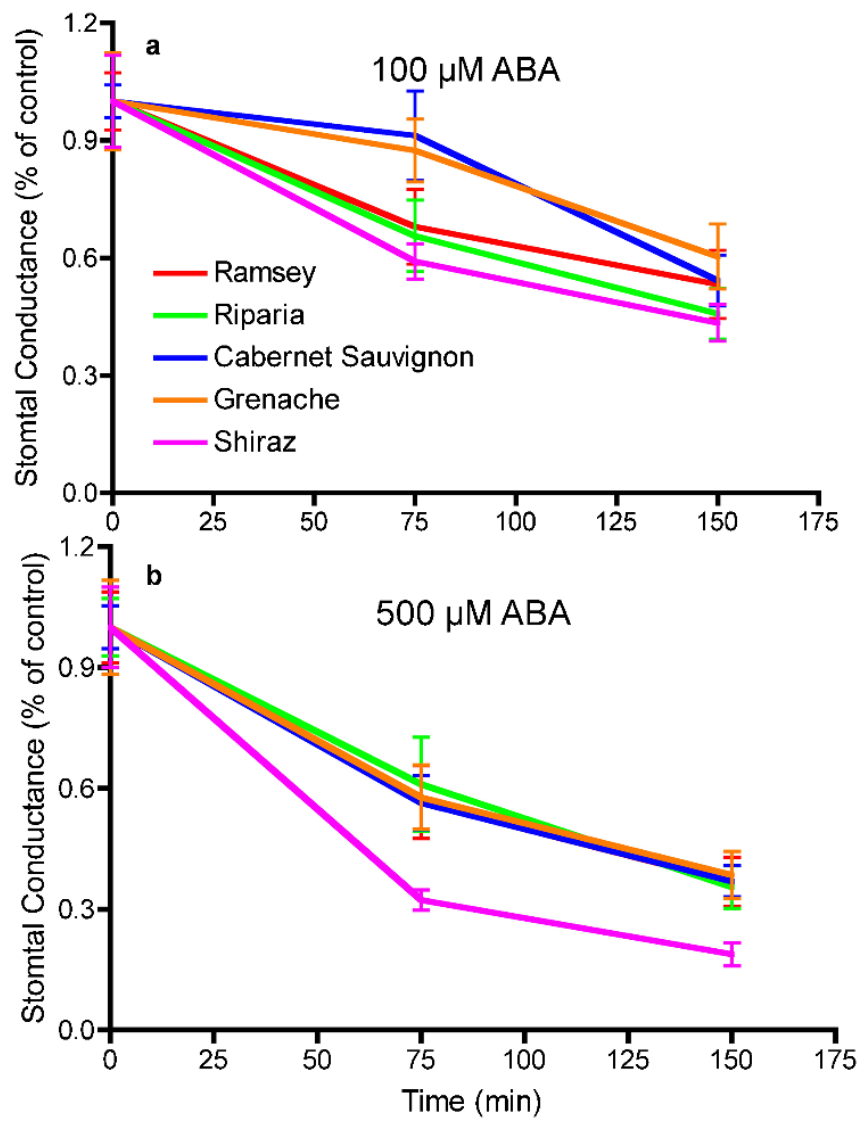

Figure 7. Stomatal sensitivities to $A B A$ of five grapevine genotypes: (a) $100 \mu \mathrm{M} \mathrm{ABA}$; (b) $500 \mu \mathrm{M}$ ABA. Data are expressed as the mean \pm s.e.m. $(n=9)$. was decreased after 75 min of treatment relative to controls. Grenache and Cabernet Sauvignon were significantly decreased relative to controls at $150 \mathrm{~min}$ after treatment, but were not significantly different from the other genotypes at this time point. Thus, Grenache and Cabernet Sauvignon responded more slowly, but quickly caught up with the other genotypes at $150 \mathrm{~min}$. The stomatal conductance of all genotypes was decreased by $500 \mu \mathrm{M} \mathrm{ABA}$ after $75 \mathrm{~min}$. Shiraz was decreased to a greater extent than all of the other genotypes at $75 \mathrm{~min}$ and $150 \mathrm{~min}$ after ABA treatment. Thus, at this $A B A$ concentration, Shiraz stomata were more sensitive to $A B A$ than the other genotypes.

\section{DISCUSSION}

Drought is the most significant abiotic stress that impacts crop production today. ${ }^{14}$ Plant scientists have developed methods for measuring plant water status for more than a century. ${ }^{2,15}$ Plants can be tested for drought tolerance by dehydration studies in the field, with potted plants, in hydroponic systems, in Petri dishes and on the bench top. Some methods are more reliable than others; some are more rapid. Yet despite this progress, there is still a call to develop better, more repeatable and quantifiable protocols for the study of drought tolerance of plants. 2,15

Drought stress experiments of grapevines are complicated, because grapevines are a woody-perennial fruit crop that can live for more than 100 years. It takes $4-6$ years for significant commercial-scale fruit production along with the establishment of a large root system.

The drought tolerance of grapevines can be associated with water use efficiency, stomatal conductance, plant hydraulic conductance, embolism repair, rooting depth and leaf dehydration tolerance. $^{3,4}$ Both hydraulic and hormonal signals (e.g., ABA) are involved in sensing water deficit and regulating plant responses. Aquaporins, which can be regulated by ABA and other plant signals, appear to be important factors that regulate water transport in the root during changes in leaf or plant water status. ${ }^{16,17}$ Nevertheless, leaves have the capacity to synthesize $A B A$ and have their own mechanisms to respond to water deficit.

There are thousands of grapevine genotypes ${ }^{18}$ and there is substantial variation in drought tolerance among the few that have been investigated and reviewed, ${ }^{3,4}$ most of which have been studied in the field. It would be very difficult to compare all known genotypes in a field study. With the rapid development of ever more powerful genotyping technologies, there is a greater need for better and more rapid phenotyping assays to be able to assess genes associated with drought tolerance, which can be used for molecular and marker-assisted plant breeding programs. ${ }^{2}$

With this goal in mind, we developed a simple and reliable rapid leaf dehydration assay. It is flexible in that it can be used on established plants in the field, greenhouse or laboratory. This assay does not require the vine to be grown in the field for $4-5$ years before testing. It can be performed on potted grapevine cuttings throughout the year if vines are grown in a greenhouse. The only limitation is the time it takes to produce fully developed leaves from potted plants.

The rationale behind using a $\mathrm{NaCl}$ solution to assess differences in dehydration response is that simply removing leaves from the plant and exposing them to the air would cause the leaves to dry out very quickly due to the strong negative water potential of the air $\left(\psi_{\text {air }}\right)$. Placing the leaf tissue within the dehydration box with a $\mathrm{NaCl}$ solution equilibrated with the air in the box, which has a calculated water potential $\left(\psi_{\mathrm{w}}\right)$ of $-0.83 \mathrm{MPa}$ at the concentration stated above, causes water to leave the leaf in a more controlled, reproducible manner down the water potential gradient. Changing the water potential of the solution and/or the temperature in the container can change the stress level. A potential error may arise when the lid of the container needs to be removed in order to weigh the 
leaf at a specific time point. However, equilibrium between the air and solution in the box will be quickly re-established when the lid is replaced. If timing is consistent, then this small error will be negligible as all leaves will be treated the same. Another possible source of variation could occur during the short time $(\sim 15 \mathrm{~s})$ from when the leaf is removed from the plant to its first weight measurement. Based on the amount of water lost at $30 \mathrm{~min}$, it can be calculated that at $15 \mathrm{~s}$, the leaves have lost $\sim 0.01 \%$ of the total weight of the leaf, which we consider insignificant. There were also no significant differences between the genotypes at $30 \mathrm{~min}$ of dehydration, which is another indication that any water lost prior to the first weight measurement is insignificant.

The normalized data (e.g., for leaf size) were consistent when the leaves were pre-selected for developmental age and healthy functions (e.g., stomatal conductance and photosynthesis). Measuring water loss from leaves was simpler and more reliable than measuring stomatal conductance with a photosynthesis system. It does not require a very expensive piece of equipment and one measures water loss from an entire leaf, not just a small patch of a leaf. Collecting data is productive because the rapid leaf dehydration assay allows an individual to measure 20 samples reliably in a period of 3 h using the only piece of sophisticated equipment required, an accurate scale. The advantages of this type of approach are that it is fast and accurate with half the variability of stomatal conductance measurements; stomatal conductance measurements had an average CV of $43.6 \%$ vs. $20.7 \%$ for leaf mass measurements (Supplementary Table 1).

Other researchers have used similar approaches to measure dehydration responses including detaching grapevine leaves and measuring their stomatal conductance in the vineyard, ${ }^{19}$ by detaching Arabidopsis leaves ${ }^{20}$ to measure stomatal conductance and water loss, or detaching Arabidopsis plants and placing them on filter paper on a bench top to measure transcriptional responses. ${ }^{21}$ In a similar approach to the assay in the present study, investigators used tissue paper that had been saturated with $1.2 \mathrm{M} \mathrm{NaCl}$, which was then placed in an enclosed container with leaves of the desiccation-tolerant plant, Borya nitidia, to investigate photosynthesis and chlorophyll kinetics. ${ }^{22}$

Stomatal response is likely controlling the kinetics of water loss in this assay. This argument is supported by similar kinetics for stomatal conductance in detached grapevine leaves in the vineyard, ${ }^{19}$ the similarities between gravimetric and stomatal conductance measurements in Arabidopsis ${ }^{20}$ and the leaf responses to ABA in the rapid leaf dehydration assay. After $4 \mathrm{~h}$ in the rapid leaf dehydration assay, water loss from the leaf was low and at a stable rate. This latter, linear phase may be caused by stomata that have failed to close or were partially open or through water loss from the cuticle of the leaf. It has been estimated that $5 \%$ of water loss by a leaf can occur through the cuticle. ${ }^{23}$

Assays comparing multiple genotypes must also consider the effects of leaf age in dehydration experiments. In previous dehydration-rehydration experiments, leaf age was an important factor in the ability of Borya nitidia to recover. ${ }^{22}$ Young leaves also act as a 'sink' leaf and may be inherently more drought tolerant because of their lack of dependence on carbon production. Under conditions of limited water, these leaves maintain a high level of stomatal conductance and may not be as sensitive to ABA. ${ }^{24}$ Older leaves would also be a poor choice for leaf selection as it has been shown that $30 \%$ of the water loss can occur through the cuticle in older Vitis vinifera leaves. ${ }^{25}$

The genotypes used in this study for the rapid leaf dehydration assay were selected based on their known differences in water deficit response. One important difference that contributed significantly to differences in water loss among the genotypes was leaf size or surface area. However, even when normalizing water loss for leaf size, differences among the genotypes were significant, indicating that other factors, such as stomatal response, contributed to the differences in water loss.

On a per leaf basis, Ramsey lost the smallest amount of water with Shiraz losing the most. These results reflect the differences in leaf size between these two genotypes as would be expected. Based on leaf size, it would be expected that Riparia Gloire would lose the most amount of water on a per leaf basis, yet this genotype falls within an intermediate group with Cabernet Sauvignon and Grenache, two genotypes with similar leaf sizes, indicating that water loss was also dependent on other factors.

Normalizing the data based on the fresh weight revealed additional information. Despite having the largest leaf size, Riparia Gloire lost the smallest amount of water compared to all other genotypes in a 3-h dehydration assay. This indicates that Riparia Gloire was the most sensitive to dehydration and responded the quickest by closing its stomata to conserve water. Shiraz, on the other hand, lost the largest amount of water, indicating that it was the least responsive to the dehydration and its stomata were slower to respond. This is consistent with the previous observations that under water deficit, Shiraz does not respond by closing its stomata and can be considered an anisohydric genotype. ${ }^{6}$ Cabernet Sauvignon lost water similarly to Shiraz, while Ramsey and Grenache lost water in a similar fashion to each other.

A number of plant variables were used in a field study to evaluate the relative drought tolerance among 17 Vitis species including pruning weight, photosynthesis, stomatal conductance, osmotic and stem water potentials. ${ }^{5} \mathrm{~V}$. champinii was ranked the most drought tolerant, $V$. vinifera was ranked in the middle and $V$. riparia was ranked as one of the most sensitive to water deficits. The drought-sensitive species, $V$. riparia, had the lowest stomatal conductance under water deficit, whereas the drought tolerant species, $V$. champinii, had the highest stomatal conductance.

Not all drought-tolerant traits are conferred by the leaves. It is clear that the root and trunk can contribute to drought tolerance as evidenced by rootstock studies. ${ }^{26-29}$ These studies support the hypothesis that Ramsey rootstock confers drought tolerance to the scion and Riparia Gloire confers drought sensitivity.

Thus, the responses of the varieties in this study with the rapid leaf dehydration assay were consistent with known grapevine genotype responses to dehydration in the field. The genotypic response is in part an inherent trait of the leaves since this response occurred in detached leaves without roots.

In this study, differences in stomatal response can be inferred from the changes in the rate of water loss over time (Figure 5c). Riparia Gloire was the quickest to respond to dehydration. Shiraz had a significantly greater rate of water loss compared to Riparia Gloire between $60 \mathrm{~min}$ and $120 \mathrm{~min}$ of dehydration. Cabernet Sauvignon had the highest initial rate of water loss, followed by a linear decrease up to $150 \mathrm{~min}$ of dehydration. Grenache and Ramsey were very similar in their rate of water loss with the greatest rate from $90 \mathrm{~min}$ to $150 \mathrm{~min}$ until leveling off at a low rate. Early differences among the genotypes in the rate of water loss were detectable at $30 \mathrm{~min}$.

Differences in the amount and rate of water loss between these genotypes indicate alternative mechanisms for water loss. One hypothesis that was tested based on the observations stated above would be that Riparia Gloire lost less water with the lowest rate of water loss compared to other genotypes due to a lower stomatal density, or fewer stomata to lose water through. The opposite could be predicted for Shiraz in that it lost the most amount of water due to a higher stomatal density. In fact, Riparia Gloire had the highest stomatal density, while Shiraz had the lowest stomatal density of the genotypes rejecting the hypothesis stated above. Ramsey and Grenache also had larger stomatal densities compared to Cabernet Sauvignon, which had a density similar to Shiraz. Despite having the lowest stomatal densities, Shiraz and Cabernet Sauvignon lost the 
most amount of water, indicating that their stomata were the least responsive to dehydration.

An alternative hypothesis is that there are differences in stomatal sensitivity to dehydration. If this were the case, one would predict that Riparia Gloire would have the highest sensitivity, Ramsey and Grenache would have an intermediate sensitivity and Shiraz and Cabernet Sauvignon would have the lowest sensitivity. This sensitivity could be affected by hydraulic and hormonal signals. ${ }^{20}$ In an early study on water deficits and $A B A,{ }^{30}$ ABA concentrations appeared to increase only after a water potential threshold was reached. In addition, differences in ABA concentrations at relatively similar water potentials among different species of plants were detected. Evidence strongly indicates that increases in drought-induced ABA concentrations in roots and leaves can affect stomatal conductance of plants and that there are interactions with other factors, such as hydraulic conductance, ethylene and cytokinins. ${ }^{31}$

$A B A$ action is dependent on $A B A$ concentrations and $A B A$ sensitivity. $A B A$ sensitivity has been associated with drought tolerance in synthetic wheats ${ }^{32}$ and stomatal behavior in Arabidopsis mutants. ${ }^{20}$ Differences in ABA sensitivity in response to dehydration have also been observed for maize genotypes differing in stressinduced ABA concentrations. ${ }^{33}$ Interestingly, the maize genotypes sensitive to $A B A$ produced lower concentrations of $A B A$ in the leaf elongation zone to achieve a decrease in leaf elongation rate compared to the insensitive genotypes, which produced a higher $A B A$ concentration to inhibit leaf elongation rate to the same extent.

Differences in leaf ABA concentrations (dependent upon ABA synthesis, degradation, conjugation and transport) and $A B A$ signaling (sensitivity) may be partially responsible for differences in water loss from the leaves in the varieties in this study. Our preliminary results indicate that some of these genotypes have different capacities to synthesize $A B A$ in response to dehydration (data not shown). Results presented here show that these genotypes do have different ABA sensitivities (Figure 7). Other studies have also detected differences in ABA concentrations in some grapevine genotypes, Grenache and Syrah, ${ }^{9,34}$ Chardonnay and Cabernet Sauvignon ${ }^{35}$ and different grapevine scion/rootstock combinations. ${ }^{28}$

Differences in ABA sensitivity cannot fully account for the different dehydration responses among these genotypes. As mentioned above, Riparia Gloire would be predicted to have the highest sensitivity to $A B A$ and Shiraz would have the lowest sensitivity to ABA. In fact, Shiraz had the highest sensitivity to $A B A$ and Riparia Gloire had an intermediate sensitivity to ABA. Other factors must also contribute to the dehydration response. It is clear that the leaf dehydration response is complicated and dependent upon multiple factors. Future research will be focused on the mechanisms of the different dehydration responses of these genotypes, including a more detailed investigation of $A B A$ action.

In summary, we developed a rapid leaf dehydration assay that could be used to detect differences among genotypes within Vitis based on their sensitivity to dehydration. The assay was simple and easy to perform in a controlled environment producing quantifiable, uniform and reliable results consistent with field observations. This assay should be amenable for use in large-scale plant phenotyping studies aimed at understanding and selecting for plant water use efficiency and drought tolerance.

\section{CONFLICT OF INTEREST}

The authors declare no conflict of interest.

\section{ACKNOWLEDGMENTS}

This work was funded by the College of Agriculture and Natural Resources at the University of Nevada, Reno with a Nevada Agriculture Experimental Station MultiState Hatch Grant (Grant No. NEV00345).

\section{REFERENCES}

1 Sirichandra C, Wasilewska A, Vlad F, Valon C, Leung J. The guard cell as a single-cell model towards understanding drought tolerance and abscisic acid action. J Exp Bot 2009; 60: 1439-1463.

2 Tuberosa R. Phenotyping for drought tolerance of crops in the genomics era. Front Physiol 2012; 3: 347.

3 Chaves MM, Zarrouk O, Francisco R et al. Grapevine under deficit irrigation: hints from physiological and molecular data. Ann Bot 2010; 105: 661-676.

4 Lovisolo C, Perrone I, Carra A et al. Drought-induced changes in development and function of grapevine (Vitis spp.) organs and in their hydraulic and non-hydraulic interactions at the whole-plant level: a physiological and molecular update. Funct Plant Biol 2010; 37: 98-116.

5 Padgett-Johnson M, Williams LE, Walker MA. Vine water relations, gas exchange, and vegetative growth of seventeen VITIS species grown under irrigated and nonirrigated conditions in California. J Am Soc Hortic Sci 2003; 128: 269-276.

6 Schultz HR. Differences in hydraulic architecture account for near-isohydric and anisohydric behaviour of two field-grown Vitis vinifera L. cultivars during drought. Plant Cell Environ 2003; 26: 1393-1405.

7 Stocker O. Die Abhängigkeit der Transpiration von den Umweltfaktoren. In: Ruhland W (ed.) Encyclopedia of PLANT PHYSIOLOGY. Berlin: Springer-Verlag, 1956.

8 Hochberg U, Degu A, Fait A, Rachmilevitch S. Near isohydric grapevine cultivar displays higher photosynthetic efficiency and photorespiration rates under drought stress as compared with near anisohydric grapevine cultivar. Physiol Plant 2013; 147: 443-452.

9 Soar CJ, Speirs J, Maffei SM, Penrose AB, McCarthy MG, Loveys BR. Grape vine varieties Shiraz and Grenache differ in their stomatal response to VPD: apparent links with ABA physiology and gene expression in leaf tissue. Aust J Grape Wine Res 2006; 12: 2-12.

10 Tardieu F, Simonneau T. Variability among species of stomatal control under fluctuating soil water status and evaporative demand: modelling isohydric and anisohydric behaviours. J Exp Bot 1998; 49(Special Issue): 419-432.

11 Lowe KM, Walker MA. Genetic linkage map of the interspecific grape rootstock cross Ramsey (Vitis champinii) $\times$ Riparia Gloire (Vitis riparia). Theor Appl Genet 2006; 112: 1582-1592.

12 Abràmoff MD, Magalhães PJ, Ram SJ. Image processing with ImageJ. Biophotonics Int 2004; 11: 36-42.

13 Chen LIN, Dodd IC, Davies WJ, Wilkinson S. Ethylene limits abscisic acid- or soil drying-induced stomatal closure in aged wheat leaves. Plant Cell Environ 2013; 36: 1850-1859.

14 Cramer GR, Urano K, Delrot S, Pezzotti M, Shinozaki K. Effects of abiotic stress on plants: a systems biology perspective. BMC Plant Biol 2011; 11: 163.

15 Jones HG. Monitoring plant and soil water status: established and novel methods revisited and their relevance to studies of drought tolerance. J Exp Bot 2007; 58: 119-130.

16 Vandeleur RK, Mayo G, Shelden MC, Gilliham M, Kaiser BN, Tyerman SD. The role of plasma membrane intrinsic protein aquaporins in water transport through roots: diurnal and drought stress responses reveal different strategies between isohydric and anisohydric cultivars of grapevine. Plant Physiol 2009; 149: 445-460.

17 Vandeleur RK, Sullivan W, Athman A et al. Rapid shoot-to-root signalling regulates root hydraulic conductance via aquaporins. Plant Cell Environ; e-pub ahead of print 8 September 2013; doi:10.1111/pce.12175.

18 This $\mathrm{P}$, Lacombe T, Thomas MR. Historical origins and genetic diversity of wine grapes. Trends Genet 2006; 22: 511-519.

19 Schultz HR. Water relations and photosynthetic responses of two grapevine cultivars of different geographical origin during water stress. Acta Hort 1996; 427: 251-266.

20 Pantin F, Monnet F, Jannaud D et al. The dual effect of abscisic acid on stomata. New Phytol 2013; 197: 65-72.

21 Yamaguchi-Shinozaki K, Shinozaki K. A novel cis-acting element in an Arabidopsis gene is involved in responsiveness to drought, low-temperature, or high-salt stress. Plant Cell 1994; 6: 251-264

22 Hethzerington S, Smillie R. Humidity-sensitive degreening and regreening of leaves of Borya nitida Labill. As followed by changes in chlorophyll fluorescence. Funct Plant Biol 1982; 9: 587-599.

23 Taiz L, Zeiger E. Plant PHYSIOLOGY. Sanderland, MA: Sinauer Associates, Inc., 2010.

24 Pantin F, Simonneau T, Muller B. Coming of leaf age: control of growth by hydraulics and metabolics during leaf ontogeny. New Phytol 2012; 196: 349-366.

25 Boyer JS, Wong SC, Farquhar GD. $\mathrm{CO}_{2}$ and water vapor exchange across leaf cuticle (epidermis) at various water potentials. Plant Physiol 1997; 114: 185-191.

26 Carbonneau A. The early selection of grapevine rootstocks for resistance to drought conditions. Am J Enol Viticult 1985; 36: 195-198.

27 McCarthy MG, Cirami RM, Furkaliev DG. Rootstock response of Shiraz (Vitis vinifera) grapevines to dry and drip-irrigated conditions. Aust J Grape Wine Res 1997; 3: 95-98. 
28 Soar CJ, Dry PR, Loveys BR. Scion photosynthesis and leaf gas exchange in Vitis vinifera L. Cv. Shiraz: mediation of rootstock effects via xylem sap ABA. Aust J Grape Wine Res 2006; 12: 82-96.

29 Stevens RM, Pech JM, Gibberd MR, Walker RR, Nicholas PR. Reduced irrigation and rootstock effects on vegetative growth, yield and its components, and leaf physiological responses of Shiraz. Aust J Grape Wine Res 2010; 16: 413-425.

30 Zabadal TJ. A water potential threshold for the increase of abscisic acid in leaves. Plant Physiol 1974; 53: 125-127.

31 Wilkinson S, Kudoyarova GR, Veselov DS, Arkhipova TN, Davies WJ. Plant hormone interactions: innovative targets for crop breeding and management. J Exp Bot 2012; 63: 3499-3509.

32 Kurahashi Y, Terashima A, Takumi S. Variation in dehydration tolerance, ABA sensitivity and related gene expression patterns in D-genome progenitor and synthetic hexaploid wheat lines. Int J Mol Sci 2009; 10: 2733-2751.
33 Cramer GR, Quarrie SA. Abscisic acid is correlated with the leaf growth inhibition of four genotypes of maize differing in their response to salinity. Funct Plant Biol 2002; 29: $111-115$.

34 Soar CJ, Speirs J, Maffei SM, Loveys BR. Gradients in stomatal conductance, xylem sap $A B A$ and bulk leaf $A B A$ along canes of Vitis vinifera cv. Shiraz: molecular and physiological studies investigating their source. Funct Plant Biol 2004; 31: 659-669.

35 Deluc L, Quilici D, Decendit A et al. Water deficit alters differentially metabolic pathways affecting important flavor and quality traits in grape berries of Cabernet Sauvignon and Chardonnay. BMC Genomics 2009; 10: 212.

(1) This work is licensed under a Creative Commons AttributionNonCommercial-NoDerivative Works 3.0 License. To view a copy of this license, visit http://creativecommons.org/licenses/by-nc-nd/3.0/

Supplemental Information for this article can be found on the Horticulture Research website (http://www.nature.com/hortres). 